\title{
DEPENDENCE OF FRIEDRICHS' CONSTANT ON BOUNDARY INTEGRALS.
}

\author{
GILES AUCHMUTY, BEHROUZ EMAMIZADEH AND MOHSEN ZIVARI
}

\begin{abstract}
This note extends the results in [2], by describing the dependence of the optimal constant in the p-version of Friedrichs' inequality on the boundary integral term. In particular, it is shown that this constant is continuous, increasing, concave and increases to the optimal constant for the Dirichlet problem as $s \rightarrow \infty$.
\end{abstract}

\section{INTRODUCTION}

Recently in [2], the optimal constants in the inequality

$$
\int_{\Omega} \sum_{j=1}^{N}\left|D_{j} u\right|^{p} d x+\int_{\partial \Omega} b|u|^{p} d \sigma \geq C_{F} \int_{\Omega} \rho|u|^{p} d x .
$$

for all $u \in W^{1, p}(\Omega)$ were studied. In particular $C_{F}$ was characterized as the principal eigenvalue of an eigenvalue problem for the p-Laplacian with Robin boundary conditions. See sections 6 and 7 of ([2]).

Here our interest is in the dependence of the constant $C_{F}$ on the boundary integral term in (1.1). Specifically we shall describe the behaviour of $C_{F}(s)$ on $[0, \infty)$ where $C_{F}(s)$ is the optimal constant in

$$
\int_{\Omega} \sum_{j=1}^{N}\left|D_{j} u\right|^{p} d x+s \int_{\partial \Omega} b|u|^{p} d \sigma \geq C_{F}(s) \int_{\Omega} \rho|u|^{p} d x .
$$

Here we shall show that $C_{F}(s)$ is increasing, locally Lipschitz continuous, and concave on $(0, \infty)$. Moreover

$$
\lim _{s \rightarrow \infty} C_{F}(s)=C_{D}
$$

where $C_{D}$ is the least eigenvalue of the Dirichlet eigenproblem for the p-Laplacian on $\Omega$. This p-Laplacian is slightly different to the usual one as studied for example in [5], but it has many similar properties and defines an equivalent norm on $W^{1, p}(\Omega)$.

\footnotetext{
Date: December 5, 2004.
} 


\section{Definitions and Notation.}

The definitions and notation of Auchmuty [2] will be used. Our essential assumptions include the following

- $\Omega$ is a non-empty bounded connected open subset of $\mathbb{R}^{N}$.

- $\partial \Omega$ is a finite union of disjoint Lipschitz surfaces with finite surface area.

- $\sigma$ represents Hausdorff $(N-1)$-dimensional surface measure on $\partial \Omega$,

We shall assume that the boundary is sufficiently regular that the Sobolev imbedding theorem and the Rellich-Kondrachov theorem hold for $W^{1, p}(\Omega)$. Specifically

(A1): The imbedding $i: W^{1, p}(\Omega) \rightarrow C^{0}(\bar{\Omega})$ is compact when $p>N$ and $i: W^{1, p}(\Omega) \rightarrow$ $L^{q}(\Omega)$ is compact for $1 \leq q<q_{c}$ when $p \leq N$ and $q_{c}=N p /(N-p)$.

Criteria for this assumption are given in Adams and Fournier [1] and in Edmunds and Evans [4] chapter V.

Let $\Gamma$ denote the boundary trace operator, then we will require (A2): The boundary trace operator $\Gamma: W^{1, p}(\Omega) \rightarrow L^{p}(\partial \Omega, d \sigma)$ is continuous.

See Evans and Gariepy [3] chapter 4 for a discussion of this.

The standard norm on $W^{1, p}(\Omega)$ is denoted $\|u\|_{1, p}$ and is defined by

$$
\|u\|_{1, p}^{p}:=\int_{\Omega}\left[\sum_{j=1}^{N}\left|D_{j} u\right|^{p}+|u|^{p}\right] d x .
$$

Our assumptions on the coefficient functions in (1.2) are (A3): The function $\rho$ is in $L^{1}(\Omega)$ when $p>N$ or else $\rho$ is in $L^{q}(\Omega)$ for some $q>q_{0}$ with $q_{0}:=N / p$ when $1<p \leq N$ and also $\rho(x) \geq \rho_{0}>0$ a.e. on $\Omega$.

(A4): $\quad b: \partial \Omega \rightarrow[0, \infty)$ is in $L^{\infty}(\partial \Omega, d \sigma)$ and $b(x)>0$ бa.e. on $\partial \Omega$.

To investigate the inequality (1.2), variational methods will be used. Define $\mathcal{F}$ : $W^{1, p}(\Omega) \times[0, \infty) \rightarrow[0, \infty)$ by

$$
\mathcal{F}(u, s):=\int_{\Omega} \sum_{j=1}^{N}\left|D_{j} u\right|^{p} d x+s \int_{\partial \Omega} b|u|^{p} d \sigma .
$$

Let $\mathcal{B}: W^{1, p}(\Omega) \rightarrow[0, \infty)$ and $\mathcal{P}: W^{1, p}(\Omega) \rightarrow[0, \infty)$ be defined by

$$
\begin{aligned}
\mathcal{B}(u) & :=\int_{\partial \Omega} b|\Gamma u|^{p} d \sigma, \quad \text { and } \\
\mathcal{P}(u) & :=\int_{\Omega} \rho(x)|u(x)|^{p} d x .
\end{aligned}
$$




\section{Description of Friedrichs' Constants}

The constant $C_{F}(s)$ in (1.2) is said to be optimal if it is the largest number such that (1.2) holds. A non-zero function $\hat{u}$ in $W^{1, p}(\Omega)$ optimizes (1.2) provided equality holds in (1.2).

When $s=0$, constant functions optimize this inequality and $C_{F}(0)=0$. Henceforth we'll consider $s \in(0, \infty)$.

The optimal constant in (1.2) can be characterized by a variational principle. Let $S_{1}:=\left\{u \in W^{1, p}(\Omega): \mathcal{P}(u)=1\right\}$. When condition (A3) holds then $S_{1}$ is a weakly closed subset of $W^{1, p}(\Omega)$ - from proposition 3.1 of [2].

Consider the family of variational principles of minimizing $\mathcal{F}(., s)$ on $S_{1}$. Then

$$
C_{F}(s):=\inf _{u \in S_{1}} \mathcal{F}(u, s)
$$

Some properties of this value function of these principles may be summarized as follows. In the following a function $g$ is said to be increasing on an interval I provided $g\left(t_{1}\right) \leq g\left(t_{2}\right)$ whenever $t_{1} \leq t_{2}$ in $\mathrm{I}$.

Theorem 3.1. Assume (A1) - (A4) hold, $1<p<\infty$ and $s \in(0, \infty)$. Then there are optimal functions $\pm u_{1}(s)$ for this variational principle. Moreover, $C_{F}(s)$ is strictly positive, increasing, locally Lipschitz and concave on $(0, \infty)$.

Proof. The existence of solutions is theorem 6.2 of [2]. In the proof of that theorem it is shown that $C_{F}(s) \in(0, \infty)$ when $s>0$. For each $u \in S_{1}, \mathcal{F}\left(u, s_{1}\right) \leq \mathcal{F}\left(u, s_{2}\right)$ whenever $s_{1}<s_{2}$, hence $C_{F}\left(s_{1}\right) \leq C_{F}\left(s_{2}\right)$.

The functionals $\mathcal{F}(u,$.$) are affine functions of s$ on $(0, \infty)$, so their infimum on $S_{1}$ will be a concave function of $\mathrm{s}$, as the infimum of any family of concave functions is concave. Since $C_{F}(s)$ is concave and finite on $(0, \infty)$ it is locally Lipschitz there.

\section{Optimal Functions as $s \rightarrow \infty$}

We now wish to prove (1.3). The optimal functions in (1.2) were characterized in section 7 of [2]. They are the non-zero functions in $W^{1, p}(\Omega)$ that satisfy

$$
\int_{\Omega}\left[\sum_{j=1}^{N}\left|D_{j} u\right|^{p-2} D_{j} u D_{j} h-\mu_{1} \rho|u|^{p-2} u h\right] d x+\int_{\partial \Omega} s b|u|^{p-2} u h d \sigma=0 .
$$


for all $h \in W^{1, p}(\Omega)$. Here $\mu_{1}$ is the least eigenvalue of this problem. This is the weak form of the p-Laplacian eigenproblem

$$
\begin{array}{ccc}
-\Delta_{p} u=-\sum_{j=1}^{N} D_{j}\left(\left|D_{j} u\right|^{p-2} D_{j} u\right) & =\mu_{1} \rho|u|^{p-2} u \quad \text { in } \Omega \\
\sum_{j=1}^{N}\left(\left|D_{j} u\right|^{p-2} D_{j} u\right) \nu_{j}+s b|u|^{p-2} u & =0 & \text { on } \partial \Omega .
\end{array}
$$

To treat the limiting case as $s$ increases, let $t:=s /(1+s)$, so this boundary condition becomes

$$
(1-t) \sum_{j=1}^{N}\left(\left|D_{j} u\right|^{p-2} D_{j} u\right) \nu_{j}+t b|u|^{p-2} u=0 \quad \text { on } \partial \Omega .
$$

Let $\mu_{1}(t)$ be the least eigenvalue of (4.1) with $s$ replaced by $t /(1-t)$ and $0 \leq t<1$ and $u_{1}(t)$ be a corresponding minimizer which exists from theorem 3.1. Then theorem 7.1 of [2] says that $\mu_{1}(t)=C_{F}(t /(1-t))$.

There is a similar variational principle for the first eigenvalue of the Dirichlet eigenproblem. Let $\mathcal{F}_{0}(u):=\mathcal{F}(u, 0)$ be defined by $(2.2)$ and $S_{0}:=\left\{u \in W_{0}^{1, p}(\Omega)\right.$ : $\mathcal{P}(u)=1\}$. Consider the variational problem of minimizing $\mathcal{F}_{0}$ on $S_{0}$ and define

$$
C_{D}:=\inf _{u \in S_{0}} \mathcal{F}_{0}(u)
$$

Just as for the previous problems, $C_{D}$ is the least eigenvalue $\hat{\mu}_{1}$ of the problem of finding nonzero functions in $W_{0}^{1, p}(\Omega)$ and eigenvalues $\mu$ satisfying

$$
\int_{\Omega} \sum_{j=1}^{N}\left|D_{j} u\right|^{p-2} D_{j} u D_{j} h d x=\mu \int_{\Omega} \rho|u|^{p-2} u h d x
$$

for all $h \in W_{0}^{1, p}(\Omega)$.

Theorem 4.1. Assume $1<p<\infty$ and (A1) - (A4) hold. Then $\lim _{t \rightarrow 1^{-}} \mu_{1}(t)=\hat{\mu_{1}}$ and (1.3) holds.

Proof. For $0 \leq t<1$ we have, since $\hat{u}_{1} \in W_{0}^{1, p}(\Omega)$,

$$
\mu_{1}(t) \leq \mathcal{F}\left(\hat{u}_{1}, t /(1-t)\right)=\hat{\mu}_{1}
$$

From theorem 3.1, $\mu_{1}(t)$ is increasing on $(0,1)$, so there is a $\mu^{*}:=\lim _{t \rightarrow 1^{-}} \mu_{1}(t)$. The preceding inequality shows that $\mu^{*} \leq \hat{\mu_{1}}$.

Let $\left\{t_{k}: k \geq 1\right\}$ be a sequence which increases to 1 and $\left\{u_{k}: k \geq 1\right\}$ be a corresponding sequence of eigenfunctions in $S_{1}$. From (4.7),

$$
0 \leq \int_{\partial \Omega} b\left|\Gamma u_{k}\right|^{p} d \sigma \leq \hat{\mu_{1}}\left(1-t_{k}\right) / t_{k}
$$


for all $k \geq 1$. Thus $\mathcal{B}\left(u_{k}\right) \rightarrow 0$ as $t_{k} \rightarrow 1^{-}$.

From (2.1), (4.7), (A3) and the definition of $S_{1}$,

$$
\left\|u_{k}\right\|_{1, p}^{p} \leq \hat{\mu}_{1}+\rho_{0}^{-1} \text { for } k \geq 1
$$

Thus this sequence has a weakly convergent subsequence, which will again be denoted $u_{k}$. Let $u^{*}$ be the weak limit of this sequence. From (A2), $\Gamma u_{k}$ converges weakly to $\Gamma u^{*}$ in $L^{p}(\partial \Omega, d \sigma)$. Thus $\mathcal{B}\left(\Gamma u^{*}\right)=0$ from (4.8) and proposition 3.2 of [2], as $\mathcal{B}$ will be weakly l.s.c. on $L^{p}(\partial \Omega, d \sigma)$. This and (A4) implies that $u^{*}=0 \sigma$ a.e. on $\partial \Omega$ or $u^{*} \in W_{0}^{1, p}(\Omega)$.

The assumption (A1) implies that $u_{k}$ converges strongly to $u^{*}$ in $L^{p}(\Omega)$ so $\mathcal{P}\left(u^{*}\right)=$ 1 and thus $u^{*} \in S_{0}$. Finally $\mathcal{F}_{0}$ is weakly l.s.c on $W^{1, p}(\Omega)$, so

$$
\mathcal{F}_{0}\left(u^{*}\right) \leq \liminf _{k \rightarrow \infty} \mathcal{F}_{0}\left(u_{k}\right) \leq \mu^{*}
$$

Thus $\hat{\mu}_{1} \leq \mu^{*}$ as $u^{*} \in S_{0}$, so $\hat{\mu_{1}}=\mu^{*}$ and the theorem is proved.

\section{REFERENCES}

[1] R.A. Adams and J.J.F. Fournier, Sobolev spaces, 2nd ed., Academic Press, 2003.

[2] G. Auchmuty, "Optimal Coercivity Inequalities in $W^{1, p}(\Omega)$ ", (submitted).

[3] L. C. Evans and R. F. Gariepy, Measure Theory and Fine Properties of Functions, CRC Press, Boca Raton (1992).

[4] D.E. Edmunds and W.D. Evans, Spectral Theory and Differential Operators, Oxford (1987).

[5] Peter Lindqvist, On the equation div $\left(|\nabla u|^{p-2} \nabla u\right)+\lambda|u|^{p-2} u=0$. Proc Amer Math Soc., 109, (1990), 157-164.

Department of Mathematics, University of Houston, Houston, TX 77204-3008, USA

Department of Mathematics, The Petroleum Institute, P. O. Box 2533, Abu DHABI, UAE.

Department of Mathematics, Iran University of Science and Technology, NarMAK, TEHRAN, IRAN

E-mail address: auchmuty@uh.edu

E-mail address: bemamizadeh@pi.ac.ae

E-mail address: m_zivari@iust.ac.ir 